\title{
The Prognostic Role of Pretreatment 18F-FDG (PET/CT) Maximum Standardized Uptake Value in Multiple or Oligometastatic Breast Cancer Patients
}

\author{
Hala A El-Lathy ${ }^{1,4}$, Ahlam A Dohal', Yasser Alassiri², Yasser Al Malki² and Ehab lbrahim ${ }^{3}$ \\ ${ }^{1}$ Department of Radiation Oncology, King Fahad Specialist Hospital, Dammam, Saudi Arabia \\ ${ }^{2}$ Department of Nuclear Medicine, King Fahad Specialist Hospital, Dammam, Saudi Arabia \\ ${ }^{3}$ Departments of Medical Oncology, King Fahad Specialist Hospital, Dammam, Saudi Arabia \\ ${ }^{4}$ Department of Clinical Oncology, Alexandria University, Egypt
}

\begin{abstract}
Purpose: To determine the prognostic role of pretreatment F-18FDG PET/CT maximum standardized uptake value (SUVmax) in metastatic breast cancer (MBC) patients and its correlation with clinicopathological parameters.

Materials and Methods: The pretreatment ${ }^{18} \mathrm{FDG}-\mathrm{PET}-\mathrm{CT}$ SUVmax in MBC patients was compared with clinicopathological parameters. The prognostic value of pretreatment SUVmax for progression free survival (PFS) and overall survival (OS) were assessed using log rank test and cox regression analysis.

Results: Overall, 100 patients $(77 \%)$ had evidence of visceral metastases while 30 patients $(23 \%)$ had oligometastatic disease confined to bones only. The receiver operator curve (ROC) demonstrated that SUVmax of 4.4 and 7.7 to be the cutoff value for predicting PFS in patients with oligometastasis to bones and multiple metastatic disease respectively. Patients with bone oligometastasis SUVmax of $>4.4$ had a significantly shorter OS [Hazard ratio (HR 3.2)] $<4.4(P<0.0001)$, whereas patients with SUV max of $\leq 4.4$ had significantly longer PFS compared with those with SUVmax $>4.4(\mathrm{P}<0.001)$. Consequently, multiple metastatic patients with SUVmax $\leq 7.7$ had significant improvement in OS compared to those with SUV max $>7.7$. On Cox regression analysis, the SUVmax category was the only factor correlated with both PFS (HR=4.5, 95\% C I 3-6.8, $\mathrm{P}<0.0001)$ and OS $(\mathrm{HR}=3.2,95 \% \mathrm{C}$ । 2.2-4.9, $\mathrm{P}<0$. 0001) in patients with multiple metastasis.
\end{abstract}

Conclusions: The pretreatment ${ }^{18}$ FDG-PET-CT SUVmax showed a statistically significant association with different clinicopathological prognostic factors. In addition, it may be considered as a potential independent prognostic indicator of clinical outcomes in metastatic breast cancer.

Keywords: ${ }^{18}$ FDG PET/CT SUVmax; Metastatic breast cancer

\section{Introduction}

Breast cancer represents a significant public health problem as it is the most common female cancer. It affects almost 1.4 million women worldwide and about 459,000 patients die due to this disease every year [1]. Approximately $6 \%$ of women with breast cancer have metastatic disease at the time of diagnosis and about $20 \%$ of patients initially diagnosed with localized disease will develop metastatic breast cancer (MBC) [2]. Despite significant improvements in the treatment of MBC during the last decade, it remains an incurable disease, with a median life expectancy of 18-30 months [3]

Hormone receptors (HR), estrogen receptor (ER) and progesterone receptor (PgR) and epidermal growth factor receptor (EGFR) play important roles in breast cancer development, progression and response to therapy. A clinically relevant subtype classification can be obtained by immunohistochemical (IHC) analysis of the tumor expression of ER, PgR, HER2 or Ki67. Five intrinsic subtypes have been identified luminal A (ER and PgR-positive, HER2-negative), luminal B (ER and/or PgR-positive, HER2-negative), luminal B like HER2positive (ER and/or PgR-positive, HER2-positive),HER2 positive ER/ PgR negative and basal type ( triple negative) [4].

Microarray gene expression analysis (cDNA) has identified these intrinsic subtypes of breast cancer. However, cDNA analysis is not routinely performed to identify breast cancer as it is too complex and costly [5].Unfortunately, these available prognostic tools in $\mathrm{MBC}$ are of limited value because of the extensive heterogeneity in breast cancer biology and variable responses to treatment like endocrine therapy, chemotherapy, and novel targeted agents. Conventional imaging procedures are commonly used to detect distant metastases with or without second primary cancers in patients with cancers, with suboptimal sensitivities and specificities [6-9].Positron emission tomography/computed tomography (PET/CT) is a widely used diagnostic tool that combines anatomic with functional imaging using $\left[{ }^{18} \mathrm{~F}\right]$-2-fluoro-2-deoxy-D-glucose (FDG), a biomarker of cellular metabolism. It can detect enhanced glycolysis of cancer cells and has proven valuable in diagnosing, staging, detecting recurrences, and assessing response to therapy in a multitude of malignant disorders [10]. The standardized uptake value (SUV) of PET/CT is a semiquantitative simplified measurement of the tissue FDG accumulation rate, and studies of the head and neck, lung, esophageal, endometrial, cervical and renal cell cancer have explored the prognostic significance of the maximum standardized uptake value (SUVmax) [11-16].

*Corresponding author: Hala A El-Lathy, MD, Department of Radiation Oncology, King Fahad Specialist Hospital, Dammam, Saudi Arabia, Tel: +966540367076; Fax: +96638412684; E-mail: h_zagloul@yahoo.com

Received June 23, 2015; Accepted July 22, 2015; Published July 27, 2015

Citation: El-Lathy HA, Dohal AA, Alassiri Y, Malki YAl, Ibrahim E (2015) The Prognostic Role of Pretreatment 18F-FDG (PET/CT) Maximum Standardized Uptake Value in Multiple or Oligometastatic Breast Cancer Patients. J Nucl Med Radiat Ther 5: 236. doi:10.4172/2155-9619.1000236

Copyright: (c) $2015 \mathrm{El}$-Lathy HA, et al. This is an open-access article distributed under the terms of the Creative Commons Attribution License, which permits unrestricted use, distribution, and reproduction in any medium, provided the original author and source are credited. 
Citation: El-Lathy HA, Dohal AA, Alassiri Y, Malki YAI, Ibrahim E (2015) The Prognostic Role of Pretreatment 18F-FDG (PET/CT) Maximum Standardized Uptake Value in Multiple or Oligometastatic Breast Cancer Patients. J Nucl Med Radiat Ther 5: 236. doi:10.4172/21559619.1000236

Moreover, the improved diagnostic performance of PET/CT imaging over conventional imaging has been investigated in the staging of high-risk patients with early breast cancer and the detection of bone metastases in patients with metastatic breast cancer [17,18]. Recently, several studies reported the correlation between maxSUV of breast cancer and several clinicopathologic or immune-histochemical features [19-23].Limitations in published series include small numbers, lack of histologic correlates, and the intra individual variation in SUV by body site and motion artifact.

Therefore, in the current retrospective, single-institution study, we examined baseline FDG avidity on PET/CT images assessed by the maximum SUV (SUVmax), by body site, as a prognostic indicator of progression free survival (PFS) and overall survival (OS) in patients with metastatic breast cancer presenting with multiple or oligometastatic disease (single/few detectable metastatic lesions) mainly to bones. Furthermore, identifying reliable prognostic markers would be of ultimate importance to individualize the management of patients with MBC (as surgical extirpation of the primary disease).

\section{Material and Method}

Retrospective review of breast cancer patients treated or referred to King Fahad Specialist Hospital-Dammam during the period between January 2010 and December 2012 after obtaining IRB approval. All patients signed informed consent. Electronic medical records were reviewed to determine known prognostic variables including: age, histology, grade, tumor phenotype (ER, PR, and HER2 expression), Ki 67 index and first-line treatment administered. Progression-free survival (PFS) was defined as the length of time from the date of the diagnosis to disease progression. Overall survival (OS) was defined as the interval between the date diagnosis and the date of death from any cause. We defined HR-positive, HER2-negative and Ki67 index $<14 \%$ as luminal A, HRpositive and HER2-positive (or HER2-negative with Ki67 index $\geq 14 \%$ ) as luminal B. Her-2/Neu status was defined positive when over-expressed with 3 plus staining in IHC or amplified with a ratio $>2.2$ by fluorescence in situ hybridization (FISH). Ki67 was visually scored for percentage of tumor cell nuclei with positive immunostaining above the background level by two pathologists.

\section{Inclusion criteria}

Female gender, 18 to70 years of age, Eastern Cooperative Oncology Group (ECOG) performance status of 0 to 2, life expectancy of $>3$ months, adequate bone marrow reserve, adequate liver and renal function, with no systemic or locoregional therapy in the metastatic setting. Biopsy proven invasive breast cancer by tru-cut biopsy, base line PET/CT as a part of staging work up, patients should have evidence of $>1$ FDG avid lesion at any of the following common metastatic breast cancer sites: bone, liver, lungs and non-regional lymph nodes.

\section{Exclusion criteria}

Patients who had excisional biopsy were excluded from the study because of higher incidence of inflammatory complications that may interfere with tumor imaging with PET/CT. In addition to patients who had received neoadjuvant chemotherapy or radiation therapy before undergoing $\mathrm{PET} / \mathrm{CT}$, brain metastasis at presentation, pregnancy or breast-feeding, history of diabetes mellitus, diagnosis of second primary malignancy, and active or uncontrolled infection.

\section{Pretreatment 18F-FDG-PET-CT scan}

The FDG-PET/CT scans were carried out using a Gemini XL PET/
CT that combines a germanium oxyorthosilicate-based PET scanner and a 16-slice Brilliance CT scanner (Philips). All patients fasted for at least 6 hours before PET scans and had serum glucoselevels $7.8 \mathrm{mmol} / \mathrm{L}$. Before and after injection, patients were kept lying comfortably in a quiet, dimly lit room. There was no significant difference in blood glucose levels measured at the time of the pre- and post-18 F-FDG studies. CT data were acquired first $(120 \mathrm{kV}, 100 \mathrm{mAs}$, no contrast enhancement). PET emission data were acquired in a 3-dimensional mode, with 3-5 min per bed position, and reconstructed using a 3-dimensional row-action maximum-likelihood algorithm.

The attenuation-corrected images were normalized for injected dose and body weight and converted into standardized uptake values (SUVs). The SUV was defined as (tracer concentration $[\mathrm{kBq} / \mathrm{mL}]$ )/ (injected activity $[\mathrm{kBq}] /$ patient body weight $[\mathrm{g}]$ ). Image acquisition was started $1 \mathrm{~h} \pm 10 \mathrm{~min}$ after intravenous administration of FDG (7.4 MBq/kg body weight). PET studies were acquired at 3-5 min per bed position, depending upon the patient's weight and body habitus, for a total of six or seven bed positions. As per our protocol, low dose CT images were obtained with oral contrast only for attenuation correction. Interpretation of the dual PET-CT images was carried out by a nuclear medicine physician/radiologist trained in PET-CT. Lesions with standardized uptake value (SUV) of $>2.5$ were considered malignant. A region of interest was drawn at each pathologic site of tracer uptake, and the SUVs were calculated automatically by the computer using the body weight method: SUV_decay-corrected activity $(\mathrm{kBq}) /$ tissue $(\mathrm{ml})$ injected FDG dose $(\mathrm{kBq}) /$ body weight $(\mathrm{g})$. Maximum SUV was measured at every site of metastases, at the primary tumor (if present), and at each of the respective regional and distant nodal groups. For patients who had multiple metastatic sites, the single lesion with the highest SUVmax was used for calculation. Tumor size had to be a minimum of $1 \mathrm{~cm}$ to minimize partial volume averaging effects in FDG-PET interpretation. For visual analysis, abnormal FDG uptake was defined as substantially greater activity in the tissue than in the aortic blood on attenuation-corrected images. When abnormal FDG uptake was present in bone, the exact anatomic location of the abnormal uptake was identified on the CT images.

\section{Statistical consideration}

The impact of different clinical parameters on Baseline SUVmax was evaluated by Mann-Whitney U test (between 2 groups) or KruskalWallis test ( $\geq 3$ groups). Receiver operator characteristic (ROC) curves were used to identify potential SUV cutoffs values in patients with multiple and oligometastatic disease. An area under the curve of 1.0 would indicate a perfect test, whereas 0.5 would represent a noninformative test. Kaplan-Meier method was accessed for survival analysis. The SUVmax values are presented as medians and interquartile ranges (IQRs), because data were not normally distributed. Prognostic variables identified by univariate analysis, with $\mathrm{P}<0.1$, were analyzed in the multivariate Cox model. All reported P-values were two-sided. Statistical significance levels were set at $\mathrm{P}<0.05$. Disease free survival (DFS) and overall survival (OS) were calculated using the Kaplan Meier analysis. Log-rank test and Cox regression analysis were performed to correlate the various clinical and pathological parameters to treatment outcomes. All analyses were performed using SPSS 16.0 package program, (SPSS, Chicago, IL).

\section{Results}

The final analysis included 130 patients (median age, 50 years; range, 29-67years) who underwent PET/CT imaging a median of 14 days before $\mathrm{MBC}$ diagnosis (range, from 28 days before to 50 days after 
Citation: El-Lathy HA, Dohal AA, Alassiri Y, Malki YAI, Ibrahim E (2015) The Prognostic Role of Pretreatment 18F-FDG (PET/CT) Maximum Standardized Uptake Value in Multiple or Oligometastatic Breast Cancer Patients. J Nucl Med Radiat Ther 5: 236. doi:10.4172/21559619.1000236

Page 3 of 7

MBC diagnosis). Other baseline characteristics are provided in Table 1. The median time from diagnosis of MBC to disease progression was 19 months (range, 11.8-36.2 months). Most patients ( $\mathrm{n}=109 ; 84 \%)$ initially had a clinically advanced stage breast cancer (stage III and IV) while the remaining 21 patients (16\%) presented with stage II disease and all patients were subsequently found to harbor metastatic disease. The vast majority of patients ( $\mathrm{n}=117 ; 90 \%)$ had invasive ductal carcinoma. With regards to tumor phenotype luminal A,B (ER/PR-positive) constituted the largest subgroup $(\mathrm{n}=64 ; 49 \%)$ whereas luminal B like, Her2neu positive and triple negative was encountered in $20 \%, 16 \%$ and $15 \%$ of the studied patient population, respectively. Thirty one patients (24\%) received endocrine therapy as their first-line treatment. Seventy-four patients (57\%) received chemotherapy, and 25 patients (19\%) received targeted therapy, possibly combined with chemotherapy or endocrine therapy. With regards to indications of PET /CT scanning as reported in patients files were: to further characterize nature of suspicious lesions detected on other radiologic studies in 65 patients $(50 \%)$ and

\begin{tabular}{|c|c|c|}
\hline Baseline Characteristic & No. of Patients & $\%$ \\
\hline \multicolumn{3}{|l|}{ Age } \\
\hline$\leq 50$ & 57 & 43 \\
\hline$>50$ & 73 & 56 \\
\hline \multicolumn{3}{|l|}{ Tumor phenotype } \\
\hline Luminal A & 30 & 23 \\
\hline Luminal B & 34 & 26 \\
\hline Luminal B like & 26 & 20 \\
\hline Her2 neu positive & 21 & 16 \\
\hline Triple negative & 19 & 15 \\
\hline \multicolumn{3}{|l|}{ Histology } \\
\hline Ductal & 117 & 90 \\
\hline Lobular & 9 & 7 \\
\hline Other & 4 & 3 \\
\hline \multicolumn{3}{|l|}{ Grade } \\
\hline 1 & 0 & \\
\hline 2 & 18 & 14 \\
\hline 3 & 112 & 86 \\
\hline \multicolumn{3}{|l|}{ Proliferation index } \\
\hline Ki $67 \% \leq 14 \%$ & 16 & 12 \\
\hline Ki $67 \% \geq 14 \%$ & 114 & 87 \\
\hline \multicolumn{3}{|c|}{ Stage at initial breast cancer diagnosis } \\
\hline Stage I & 0 & \\
\hline Stage II & 21 & 16 \\
\hline Stage III & 46 & 35 \\
\hline Sage IV & 63 & 49 \\
\hline \multicolumn{3}{|l|}{ Indications for PET/CT } \\
\hline Other abnormal radiology & 65 & 50 \\
\hline Locally advanced breast cancer & 23 & 18 \\
\hline Symptoms & 32 & 25 \\
\hline Indication not determined & 10 & 7 \\
\hline \multicolumn{3}{|l|}{ First therapy for MBC } \\
\hline Endocrine therapy & 31 & 24 \\
\hline $\begin{array}{l}\text { Targeted with or without } \\
\text { endocrine therapy }\end{array}$ & 25 & 19 \\
\hline Chemotherapy & 74 & 57 \\
\hline
\end{tabular}

Table 1: Baseline characteristics of the studied group of patients. to assess patients presenting with either locally advanced breast cancer or with symptoms in (18\%) and (25\%) of patients respectively (Table 2). Overall, 100 patients (77\%) had evidence of visceral with or without non regional lymph node metastases (defined as lung 38 patients (29\%) or liver in 37 patients (28\%) disease and nonregional lymph node metastasis 25 patients (19\%) on PET/CT images). Moreover, bones as oligometastatic site, were observed in 30 patients $(23 \%)$ of the studied population. In total, 110 patients $(85 \%)$ had at least 1 biopsy result that confirmed the MBC diagnosis. Among the patients with FDG-avid lesions, according to anatomic site, the numbers with positive biopsies were as follows: bones, $250 \mathrm{of} 30$ patients $(83 \%)$; liver, 33 of 37 patients (89\%); LN, 23of 25 patients (92\%); and lung, 29 of 38 patients (76\%). The median SUVmax of the studied 130 patients was $13.3 \pm 6.1$ (range, 2.8-24.3). Median SUVmax was also significantly different among different tumor grade groups $(\mathrm{P}<0.001)$ and was increased by increases in the tumor grade. The SUVmax was significantly higher in triple negative tumors $(\mathrm{P}<0.001)$ and Her2neu positive tumors $(\mathrm{P}=0.03)$, compared to luminal A,B tumors respectively (Table 3 ).

In patient presented with breast cancer metastasizing to bone only, the receiver operator curve (ROC) demonstrated a SUVmax of 4.4 to be the optimal cutoff for predicting PFS (area under the curve: 0.681; standard error: 0.0678 ). A SUVmax of 4.4 yielded a sensitivity of $68.7 \%$ and a specificity of $78.5 \%$ for predicting the PFS (Figure 1). Similarly, patient presented with multiple metastatic disease, the receiver operator curve (ROC) demonstrated a SUVmax of 7.7 to be the optimal cutoff for predicting PFS (area under the curve: 0.837; standard error: 0.058). A SUVmax of 7.7 yielded a sensitivity of $88 \%$ and a specificity of $82.5 \%$ for predicting the PFS (Figure 2).

\section{Standard prognostic variables}

We first examined known prognostic variables (intrinsic phenotype, metastatic disease site, first line treatment, age, tumor grade and histology) for the whole cohort irrespective of disease site and demonstrated the inferior OS of patients with triple-negative disease (negative for ER, PR, and HER2; HR, 2.8) compared with luminal $\mathrm{A}, \mathrm{B}(\mathrm{ER} / \mathrm{PR}$-positive and HER2-negative disease $)(\mathrm{P}<0.01)$ (Figure 3). Similarly, patients who had visceral metastases $(\mathrm{N}=75)$ had inferior survival $(\mathrm{HR}, 1.6 ; \mathrm{P}=0.04)$ compared with patients who did not. Patients who received targeted therapy (including with endocrine therapy or chemotherapy) or chemotherapy alone in the first-line setting had significantly decreased survival $(\mathrm{P}=0.002$; $\mathrm{HR}, 1.8$ and 3.6, respectively) compared with patients who received endocrine therapy. It is noteworthy that grade $(\mathrm{P}=0.08)$, age $(\mathrm{P}=0.35)$, and histologic subtype $(\mathrm{P}=0.75)$ had no significant effect on prognosis.

\section{Maximum standard uptake value as a prognostic variable}

A strong correlation between the SUVmax cut off value 4.4 in bone and OS was observed in the survival analysis using the KaplanMeier method. As patients with a SUVmax of more than 4.4 had a significantly shorter OS (HR, 3.2) than patients with less than 4.4 $(\mathrm{P}<0.0001)$ (Figure 4). The magnitude of this effect was smaller than the effect of other variables, such as triple- negative histology (HR, 4.16; $\mathrm{P}<0.001)$. Furthermore, patients with bone metastasis having SUV max of 4.4 or less median progression free was not reached, consequently they had significantly longer progression free survival compared to patients with more than 4.4 in their bone metastasis $(\mathrm{P}<0.001)$ (Figure 5). Additionally, it was observed that patient presenting with multiple metastasis with SUV max cut off $\leq 7.7$ had significant improvement in OS (HR, 2.2) and PFS (HR, 2.5) compared to those patients with SUV max cut off value $>7.7$ (Figure 6). On multivariate analysis, the 
Citation: El-Lathy HA, Dohal AA, Alassiri Y, Malki YAI, Ibrahim E (2015) The Prognostic Role of Pretreatment 18F-FDG (PET/CT) Maximum Standardized Uptake Value in Multiple or Oligometastatic Breast Cancer Patients. J Nucl Med Radiat Ther 5: 236. doi:10.4172/21559619.1000236

Page 4 of 7

Table 2: Maximum standardized uptake value by disease site.

\begin{tabular}{|l|c|}
\hline Disease Site & SUV max Values \\
\hline Bone N=30 & 4 \\
\hline Median & $5.2(2.8-8)$ \\
\hline Range & 3 \\
\hline Low quartile & 5 \\
\hline High quartile & 13.4 \\
\hline Liver N=37 & $17.8(5.2-23)$ \\
\hline Median & 8.9 \\
\hline Range & 19.1 \\
\hline Low quartile & 8.9 \\
\hline High quartile & $12.7(5.3-18)$ \\
\hline Lung N=38 & 6.7 \\
\hline Median & 17.4 \\
\hline Range & \\
\hline Low quartile & 10 \\
\hline High quartile & $13.4(5.1-18.5)$ \\
\hline Lymph node $\mathrm{N}=25$ & 7 \\
\hline Median & 17 \\
\hline Range & \\
\hline Low quartile & \\
\hline High quartile & \\
\hline
\end{tabular}

Table 3: Baseline SUVmax comparison between and among groups.

\begin{tabular}{|c|c|c|c|c|}
\hline \multirow{2}{*}{ Baseline Characteristic } & \multirow{2}{*}{ No. of Patients } & \multirow{2}{*}{$\%$} & \multicolumn{2}{|c|}{ Baseline SUVmax } \\
\hline & & & Median & $P$ value \\
\hline \multicolumn{5}{|l|}{ Age } \\
\hline$\leq 50$ & 57 & 43 & 6.3 & 0.456 \\
\hline$>50$ & 73 & 56 & 6.9 & \\
\hline \multicolumn{5}{|l|}{ Tumor phenotype } \\
\hline Luminal A & 30 & 23 & 5.7 & 0.234 \\
\hline Luminal B & 34 & 26 & 6.8 & 0.544 \\
\hline Luminal B like & 26 & 20 & 7.9 & 0.136 \\
\hline Her2 neu positive & 21 & 16 & 9.2 & $0.03^{*}$ \\
\hline Triple negative & 19 & 15 & 11.8 & $<0.001^{*}$ \\
\hline \multicolumn{5}{|l|}{ Histology } \\
\hline Ductal & 117 & 90 & 6.5 & \\
\hline Lobular & 9 & 7 & 5.8 & 0.453 \\
\hline Other & 4 & 3 & 7.8 & \\
\hline \multicolumn{5}{|l|}{ Grade } \\
\hline 1 & 0 & & & \\
\hline 2 & 18 & 14 & 7 & \\
\hline 3 & 112 & 86 & 9.8 & $<0.001^{*}$ \\
\hline \multicolumn{5}{|l|}{ Proliferation index } \\
\hline Ki $67 \% \leq 14 \%$ & 16 & 12 & 4.8 & \\
\hline Ki $67 \% \geq 14 \%$ & 114 & 87 & 9.8 & $<0.001^{*}$ \\
\hline \multicolumn{5}{|l|}{ Metastatic sites } \\
\hline Visceral & 100 & 77 & 10 & \\
\hline Bone only & 30 & 23 & 4 & $0.005^{*}$ \\
\hline \multicolumn{5}{|l|}{ No. of metastatic sites } \\
\hline 1 & 49 & 38 & 4 & \\
\hline 2 & 30 & 23 & 6.8 & $0.003^{*}$ \\
\hline$\geq 3$ & 51 & 39 & 9.8 & \\
\hline \multicolumn{5}{|l|}{ First therapy for MBC } \\
\hline Endocrine therapy & 31 & 24 & 4 & \\
\hline $\begin{array}{l}\text { Targeted } \pm \text { endocrine } \\
\text { therapy }\end{array}$ & 25 & 19 & 6.6 & 0.643 \\
\hline Chemotherapy & 74 & 57 & 8.7 & \\
\hline
\end{tabular}

SUVmax category was the only factor associated with PFS $(\mathrm{HR})=8.4$, 95\% CI 1.2-54.8, $\mathrm{P}<0.026)$ but not $\mathrm{OS}(\mathrm{P}=0.99)$ in oligometastatic patients to bones only. Additionally, the SUVmax category was the only factor correlated with both PFS (Hazard ratio (HR) $=4.5,95 \% \mathrm{C}$ I 3-6.8, $\mathrm{P}<0.0001)$ and $\mathrm{OS}(\mathrm{HR}=3.2,95 \% \mathrm{C}$ I $2.2-4.9, \mathrm{P}<0.0001)$ in multiple metastatic patients.

\section{Discussion}

Various pathological prognostic indicators such as intrinsic phenotypes, metastatic disease sites, first line treatment, age, tumor grade and histology have been proposed for the risk stratification of MBC patients but they can only be obtained after surgery. On the other hand, $\mathrm{F}-{ }^{18} \mathrm{FDG}$ PET can provide quantitative information about tumor glucose metabolism, which represents the aggressiveness of the malignant lesion. FDG uptake can be evaluated noninvasively and be measured with good inter-test reproducibility [24].

In the present study, 100 patients (77\%) had evidence of visceral metastases with or without non regional lymph node involvement. While oligometastatsis to bones only, were observed in 30 patients

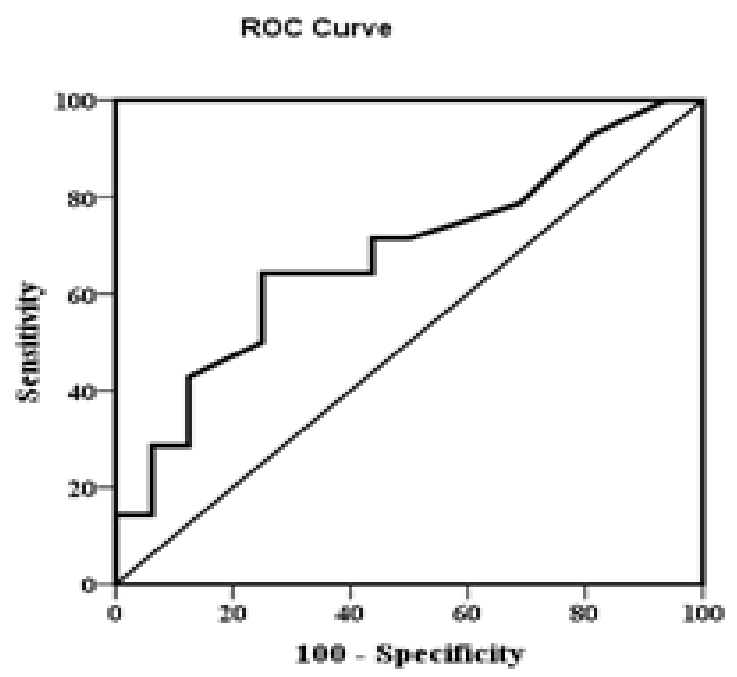

Figure 1: The receiver operator curve (ROC) in breast cancer metastasizing to bone only.

\section{ROC Curve}

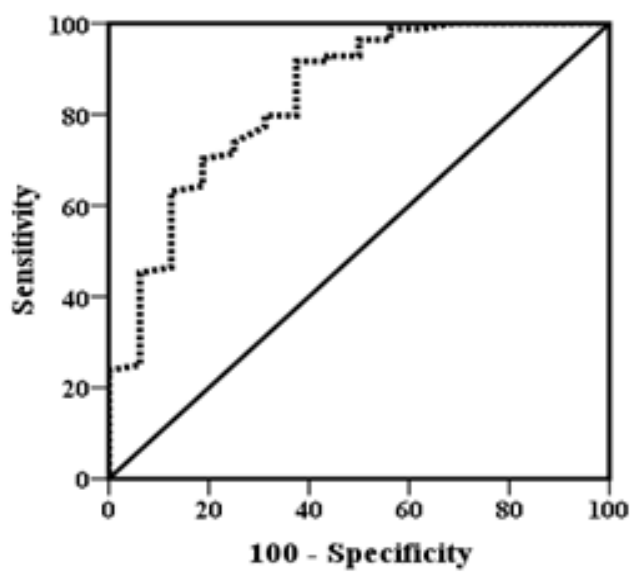

Figure 2: The receiver operator curve (ROC) in multiple metastatic breast cancer patients. 
Citation: El-Lathy HA, Dohal AA, Alassiri Y, Malki YAI, Ibrahim E (2015) The Prognostic Role of Pretreatment 18F-FDG (PET/CT) Maximum Standardized Uptake Value in Multiple or Oligometastatic Breast Cancer Patients. J Nucl Med Radiat Ther 5: 236. doi:10.4172/21559619.1000236

Page 5 of 7

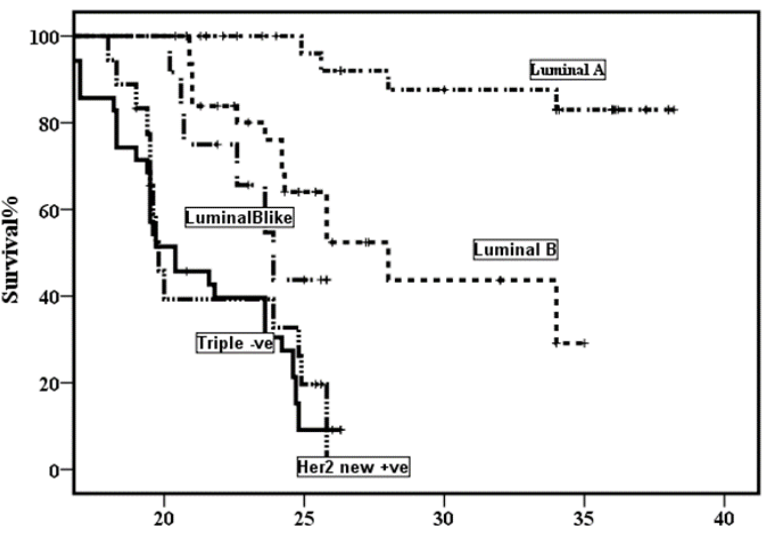

Figure 3: Overall survival of different phenotypes.

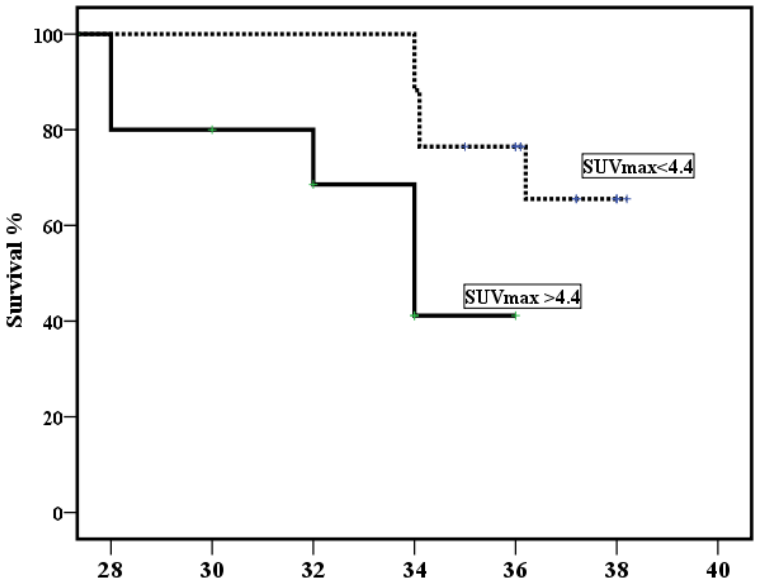

Figure 4: Overall survival for patients with bone metastasis.

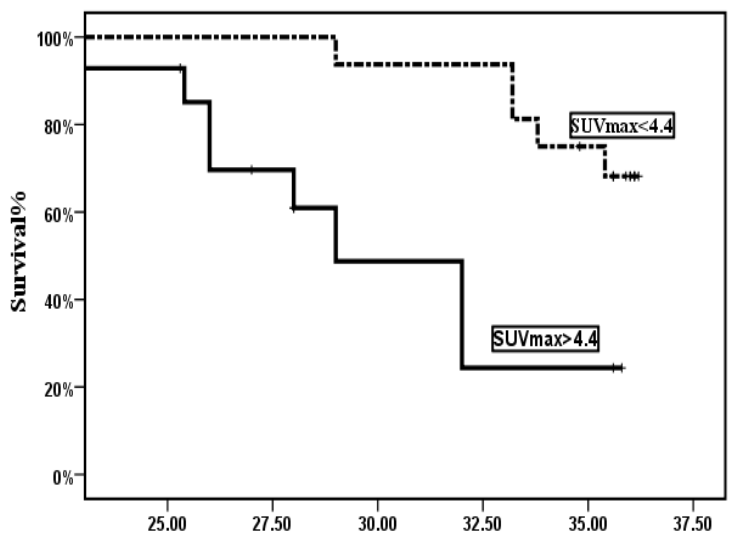

Figure 5: Progression free survival for patients with bone metastasis.

(23\%). In total, 110 patients (85\%) had at least 1 biopsy result that confirmed the metastatic breast cancer diagnosis. The SUVmax was significantly higher in triple negative tumors $(\mathrm{P}<0.001)$ and Her2 neu positive tumors $(\mathrm{P}=0.03)$ compared to luminal $\mathrm{A}$ and $\mathrm{B}$ tumors respectively. Kim et al. also reported that triple negative tumors had a significantly higher maxSUV than non-triple negative tumors $(\mathrm{p}=0.016)$ [25]. Similarly, Basu et al. observed that triple negative breast tumors were associated with enhanced FDG uptake commensurate with their aggressive biology [19].

On univariate analysis, there was inferior OS for patients with triple-negative disease (HR 2.8) compared with luminal A and B (ER/ $\mathrm{PR}$-positive and HER2-negative disease) $(\mathrm{P}<0.01)$. Moreover, patients who had visceral metastases $(\mathrm{N}=100)$ had inferior survival $(\mathrm{HR}, 1.6$; $\mathrm{P}=0.04)$ compared to patients who did not. Zhang et al. also reported that the presence of visceral metastasis $(\mathrm{P}=0.035)$, number of metastatic sites $(\mathrm{P}=0.002)$, chemotherapy as the first-line therapy after PET/CT $(\mathrm{P}=0.037)$ were significantly associated with shorter PFS and OS [26]. It is noteworthy that: grade $(\mathrm{P}=0.08)$, age $(\mathrm{P}=0.35)$, histologic subtype $(\mathrm{P}=0.75)$ had no significant effect on prognosis. Morris et al reported comparable results, as grade $(\mathrm{P}=0.09)$, age $(\mathrm{P}=0.45)$, histologic subtype $(\mathrm{P}=0.95)$ were found to have no significant impact on survival [27].

To the best of our knowledge, this retrospective study represents the first series that succeeded to find out different cut off values of baseline 18 F PET-CT FDG SUVmax uptake for breast cancer patients presenting with multiple visceral or oligometastasis to bones (7.7 and 4.4) respectively. In the current study, patients presenting with multiple metastases with SUV max cut off $\leq 7.7$ had significant improvement in OS (HR, 2.2) and PFS (HR, 2.5) compared to those patients with SUV max cut off value $>7.7$. On multivariate analysis, the SUVmax category was the only factor correlated with both PFS (Hazard ratio $(\mathrm{HR})=4.5$, 95\% C I 3-6.8, P<0. 0001) and OS ( HR=3.2, 95\% C I 2.2-4.9, P<0.0001). Furthermore, these SUVmax cut off values yielded a sensitivity of $88 \%$ and a specificity of $82.5 \%$ for prediction the PFS in patients presenting with multiple visceral metastases. This finding was also confirmed previously in other trials $[28,29]$. Bong et al. reported a cut off value of 6.6 for the SUVmax for the whole group without segregation of patients according to the site of metastasis (visceral or bone) and he also demonstrated longer survival in patients with a lower SUV [28].

Additionally, patients with bone metastasis having SUVmax of $>4.4$ had a significantly shorter OS (HR 3.2) than patients with $<4.4$ $(\mathrm{P}<0.0001)$. Moreover, the SUVmax cutoff value of 4.4 in oligometastasis to bones achieved sensitivity and specificity in predicting PFS of $68.7 \%$ and $78.5 \%$ respectively. Correspondingly, Morris et al observed a strong correlation between the SUVmax in bone and OS $(\mathrm{P}<0.001)$. By using the tertile with the lowest SUVmax as the reference group (median, 4.7;

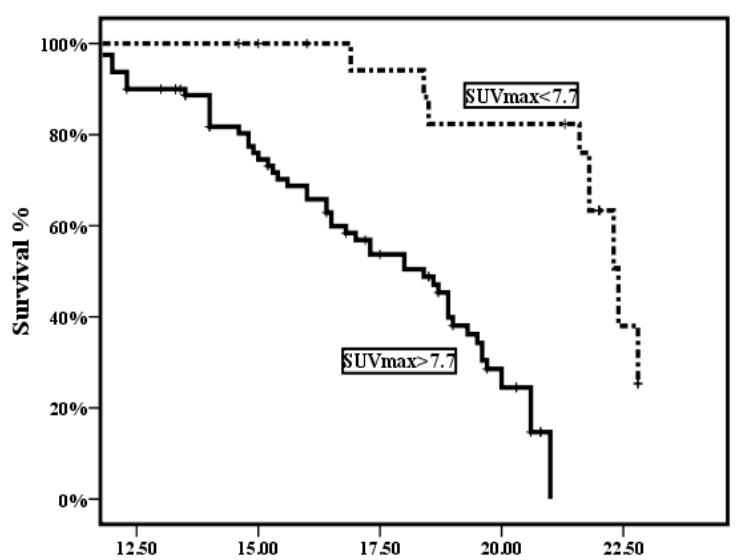

Figure 6: Progression free survival for patients with multiple visceral metastases. 
Citation: El-Lathy HA, Dohal AA, Alassiri Y, Malki YAI, Ibrahim E (2015) The Prognostic Role of Pretreatment 18F-FDG (PET/CT) Maximum Standardized Uptake Value in Multiple or Oligometastatic Breast Cancer Patients. J Nucl Med Radiat Ther 5: 236. doi:10.4172/21559619.1000236

Page 6 of 7

range, 2.1-5.8), patients in the highest tertile of SUVmax (median, 11.2, range, 9.3-29.6) had the shortest survival (HR, 3.13) [27].

\section{The current study has several strengths}

First: it included a broad representation of various intrinsic subgroups of breast cancer.

Second: 110 patients (85\%) had at least 1 biopsy result that confirmed the MBC diagnosis (the gold standard), which contrasts to some other series in which the diagnostic performance of PET/CT imaging was compared with other imaging modalities.

Third: we correlated FDG uptake (SUVmax) with OS, which is a clean endpoint, as this considers both variable tumor biology and treatment administered.

There are limitations to the current study: it was retrospective, it did not assess tumor: background ratios, it included a heterogeneous population both in terms of variable follow up imaging (timing and modality) and treatment regimens administered. Although $85 \%$ of patients underwent a biopsy of at least 1 site, we cannot be absolutely sure that all of the FDG-avid lesions observed on PET/CT images truly represented MBC. Furthermore, we examined PET/CT imaging from only 1 time-point and thus are unable to comment on the prognostic effect of PET/CT imaging (with regard to treatment effect). Finally, because this was a retrospective study, the cost-effectiveness of PET/ CT imaging could not be assessed.

\section{Conclusion}

This study demonstrates that the pretreatment 18FDG-PET-CT SUVmax showed a statistically significant association with different clinicopathological prognostic factors. In addition, it may be considered as a potential independent prognostic indicator of clinical outcomes in metastatic breast cancer patients.Ultimately prospective studies will be needed to further validate the prognostic potential of pretreatment 18FDG-PET-CT SUVmax in MBC patients.

\section{Conflict of Interest}

All authors confirm that there is no conflict of interest and they all agree to the manuscript. No financial support nor grants were offered to this research.

\section{References}

1. Youlden DR, Cramb SM, Dunn NA, Muller JM, Pyke CM, et al. (2012) The descriptive epidemiology of female breast cancer: an international comparison of screening, incidence, survival and mortality. Cancer Epidemiol 36: 237-248.

2. Brewster AM, Hortobagyi GN, Broglio KR, Kau SW, Santa-Maria CA, et al. (2008) Residual risk of breast cancer recurrence 5 years after adjuvant therapy. J Natl Cancer Inst 100: 1179-1183.

3. Mariani G (2005) New developments in the treatment of metastatic breast cancer: from chemotherapy to biological therapy. Ann Oncol 16 Suppl 2: ii191-194.

4. Carey LA, Perou CM, Livasy CA, Dressler LG, Cowan D, et al. (2006) Race breast cancer subtypes, and survival in the Carolina Breast Cancer Study. JAMA 295: 2492-2502.

5. Sørlie T, Perou CM, Tibshirani R, Aas T, Geisler S, et al. (2001) Gene expression patterns of breast carcinomas distinguish tumor subclasses with clinical implications. Proc Natl Acad Sci U S A 98: 10869-10874.

6. Ng SH, Chan SC, Yen TC, Chang JT, Liao CT, et al. (2009) Staging of untreated nasopharyngeal carcinoma with $\mathrm{PET} / \mathrm{CT}$ : comparison with conventional imaging work-up. Eur J Nucl Med Mol Imaging 36: 12-22.

7. $\mathrm{Ng} \mathrm{SH}$, Chan SC, Liao CT, Chang JT, Ko SF, et al. (2008) Distant metastases and synchronous second primary tumors in patients with newly diagnosed oropharyngeal and hypopharyngeal carcinomas: evaluation of 18F-FDG PET and extended-field multidetector row CT. Neuroradiology 50: 969-979.

8. Stroobants SG, D'Hoore I, Dooms C, De Leyn PR, Dupont PJ, et al. (2003) Additional value of whole-body fluorodeoxyglucose positron emission tomography in the detection of distant metastases of non-small-cell lung cancer. Clin Lung Cancer 4: 242-247.

9. Niikura N, Costelloe CM, Madewell JE, Hayashi N, Yu TK, et al. (2011) FDG$\mathrm{PET} / \mathrm{CT}$ compared with conventional imaging in the detection of distant metastases of primary breast cancer. Oncologist 16: 1111-1119.

10. Alavi A, Lakhani P, Mavi A, Kung JW, Zhuang H (2004) PET: a revolution in medical imaging. Radiol Clin North Am 42: 983-100, vii.

11. Allal AS, Slosman DO, Kebdani T, Allaoua M, Lehmann W, et al. (2004) Prediction of outcome in head-and-neck cancer patients using the standardized uptake value of 2-[18F]fluoro-2-deoxy-D-glucose. Int J Radiat Oncol Biol Phys 59: $1295-1300$.

12. Downey RJ, Akhurst T, Gonen M, Vincent A, Bains MS, et al. (2004) Preoperative F-18 fluorodeoxyglucose-positron emission tomography maximal standardized uptake value predicts survival after lung cancer resection. J Clin Oncol 22: 3255-3260.

13. Sasaki R, Komaki R, Macapinlac H, Erasmus J, Allen P, et al. (2005) [18F] fluorodeoxyglucose uptake by positron emission tomography predicts outcome of non-small-cell lung cancer. J Clin Oncol 23: 1136-1143.

14. Kitajima K, Kita M, Suzuki K, Senda M, Nakamoto Y, et al. (2012) Prognostic significance of SUVmax (maximum standardized uptake value) measured by [(18)F]FDG PET/CT in endometrial cancer. Eur J Nucl Med Mol Imaging 39: 840-845.

15. Lee YY, Choi $\mathrm{CH}$, Kim CJ, Kang H, Kim TJ, et al. (2009) The prognostic significance of the SUVmax (maximum standardized uptake value for F-18 fluorodeoxyglucose) of the cervical tumor in PET imaging for early cervical cancer: preliminary results. Gynecol Oncol 115: 65-68.

16. Namura K, Minamimoto R, Yao M, Makiyama K, Murakami T, et al. (2010) Impac of maximum standardized uptake value (SUVmax) evaluated by 18-Fluoro-2deoxy-D-glucose positron emission tomography/computed tomography (18 F-FDG-PET/ CT) on survival for patients with advanced renal cell carcinoma: a preliminary report. BMC Cancer 10: 667.

17. Berriolo-Riedinger A, Touzery C, Riedinger JM, Toubeau M, Coudert B, et al. (2007) [18F]FDG-PET predicts complete pathological response of breast cancer to neoadjuvant chemotherapy. Eur J Nucl Med Mol Imaging 34: 1915-1924.

18. Groheux D, Giacchetti S, Rubello D, Al-Nahhas A, Moretti JL, et al. (2010) The evolving role of PET/CT in breast cancer. Nucl Med Commun 31: 271-273.

19. Basu S, Chen W, Tchou J, Mavi A, Cermik T, et al. (2008) Comparison of triplenegative and estrogen receptor-positive/ progesterone receptor-positive/HER2 negative breast carcinoma using quantitative fluorine-18 fluorodeoxyglucose/ positron emission tomography imaging parameters: a potentially useful method for disease characterization. Cancer 112: 995-1000.

20. Groheux D, Giacchetti S, Moretti JL, Porcher R, Espie M, et al. (2011) Correlation of high 18F-FDG uptake to clinical, pathological and biological prognostic factors in breast cancer. Eur J Nucl Med Mol Imaging 38: 426-435.

21. Heusner TA, Kuemmel S, Hahn S, Koeninger A, Otterbach F, et al. (2009) Diagnostic value of full-dose FDG PET/CT for axillary lymph node staging in breast cancer patients. Eur J Nucl Med Mol Imaging 36: 1543-1550.

22. Ueda S, Tsuda H, Asakawa H, Shigekawa T, Fukatsu K, et al. (2008) Clinicopathological and prognostic relevance of uptake level using 18F-fluorodeoxyglucose positron emission tomography/ computed tomography fusion imaging (18F-FDG PET/CT) in primary breast cancer. Jpn J Clin Oncol 38: $250-258$

23. Veronesi U, De Cicco C, Galimberti VE, Fernandez JR, Rotmensz N, et al. (2007) A comparative study on the value of FDG-PET and sentinel node biopsy to identify occult axillary metastases. Ann Oncol 18: 473-478.

24. Minn H, Zasadny KR, Quint LE, Wahl RL (1995) Lung cancer: reproducibility of quantitative measurements for evaluating 2-[F-18]-fluoro-2-deoxy-D-glucose uptake at PET. Radiology 196: 167-173.

25. Kim BS, Sung SH (2012) Usefulness of 18F-FDG uptake with clinicopathologic and immunohistochemical prognostic factors in breast cancer. Ann Nucl Med 26: $175-183$ 
Citation: El-Lathy HA, Dohal AA, Alassiri Y, Malki YAI, Ibrahim E (2015) The Prognostic Role of Pretreatment 18F-FDG (PET/CT) Maximum Standardized Uptake Value in Multiple or Oligometastatic Breast Cancer Patients. J Nucl Med Radiat Ther 5: 236. doi:10.4172/21559619.1000236

26. Zhang J, Jia Z, Ragaz J, Zhang YJ, Zhou M, et al. (2013) The maximum standardized uptake value of 18 F-FDG PET scan to determine prognosis of hormone-receptor positive metastatic breast cancer. BMC Cancer 13: 42.

27. Patrick GM, Gary AU, Anne E, Fazio M, Jhaveri K, et al. (2012) Standardized Uptake Value by Positron Emission Tomography/Computed Tomography as A Prognostic Variable in Metastatic Breast Cancer. Cancer 118: 5454-5462.
28. Song $\mathrm{BI}$, Hong CM, Lee HJ, Kang S, Jeong SY, et al. (2011) Prognostic Value of Primary Tumor Uptake on F-18 FDG PET/CT in Patients with Invasive Ducta Breast Cancer. Nucl Med Mol Imaging 45: 117-124.

29. Oshida M, Uno K, Suzuki M, Nagashima T, Hashimoto H, et al. (1998) Predicting the prognoses of breast carcinoma patients with positron emission tomography using 2-deoxy-2-fluoro[18F]-D-glucose. Cancer 82: 2227-2234. 